\title{
Design and Implementation of Intelligent Wheelchair System Based on EEG Control
}

\author{
Hongsen Zhou ${ }^{1, a}$ and Guolong Zhang ${ }^{1, b, *}$
}

\author{
${ }^{I}$ School of Computer Science, Jiangxi University of Traditional Chinese Medicine, Nanchang, Jiangxi, China \\ zhouhongsen5.12@foxmail.com, ${ }^{b} 65168700 @ q q . c o m$ \\ *Corresponding author
}

\begin{abstract}
In order to improve the self-care ability of disabled people, this paper designs and implements an intelligent wheelchair system based on EEG control. The human EEG signal collected by the sensor is sent to the core control board through Bluetooth device. And the wavelet packet transform is used for noise reduction and feature extraction. Then the multi-class SVM technology is used to recognize the EEG signal and realize the intelligent control of the wheelchair in various motion states. In the experiment, the average Kappa coefficient reach 0.622, which is better than BP neural network. Moreover, the system has short response time and high recognition rate, which further showing the effectiveness and reliability of the proposed method.
\end{abstract}

Keywords: EEG, intelligent wheelchair, Wavenet De-nosing, feature-extraction, multi-class SVM

\section{基于脑电波控制的智能轮椅系统设计与实现}

\author{
周宏森 ${ }^{1, \mathrm{a}}$, 张国龙 ${ }^{1, \mathrm{~b}, *}$
}

\author{
${ }^{I}$ 计算机学院, 江西中医药大学, 南昌, 江西, 中国 \\ ${ }^{a}$ zhouhongsen5.12@foxmail.com, ${ }^{b} 65168700 @ q q . c o m$ \\ *通讯作者
}

\section{摘要}

为了改善残障人士的生活自理能力，本文设计并实现了一种基于脑电波控制的智能轮椅系统。将传感 器采集到的人体脑电信号通过蓝牙设备发送到核心控制板，应用小波包变换进行降噪和特征提取，然 后采用多分类 SVM 技术对脑电波信号进行模式识别, 实现了轮椅多种运动状态的智能控制。实验的平 均 Kappa 系数达到了 0.622 , 效果优于 BP 神经网络, 并且该系统响应时间短, 识别率高, 进一步表明 所提方法的有效性和可靠性。

关键词：脑电信号，智能轮椅，小波消噪，特征提取，多分类支持向量机

\section{1. 引言}

智能轮椅 [1-4]作为移动机器人的一种, 有助于改善老年人 和残疾人的生活质量、让他们重获自理能力, 其研究也得 到越来越多的关注 $[5]$ 。近年来, 利用高性能的人体脑电 波信号控制智能轮椅[6]已成为生命科学和工程学领域的 研究热点。脑电信号 (EEG) 是脑神经细胞群电生理活动 的综合反映, 其中蕴涵着丰富的生理、心理信息。通过脑 电信号分析, 解读出操作者的意愿 [7], 获得相应的控制 信息, 能够有效控制外部对象 [8]。基于此, 本文提出一 种利用人体脑电波信号来控制智能轮椅运动轨迹的设计 方法。经过实践发现采用大脑运动想象的意念就可以很好
地控制电动轮椅的前进、停止、后退、左转、右转等运动 轨迹, 同时还具有基本的环境监测功能。具有脑电波信号 控制的智能轮椅从一个崭新的角度出发, 综合生物医学、 计算机、控制理论等各领域, 能够为肢体有残疾的群体提 供更加人性化、智能化的帮助[9-10]。

\section{2. 系统方案设计}

脑电波信号是一类重要的微弱生物电信号, 可以在一定程 度上反应人体行为意图[11]。本文通过设计相应的脑电信 号控制轮椅的方案, 对脑电信号进行采集和预处理, 识别 多种动作意念, 并将识别到的动作意图编码成控制指令, 最终实现对电动轮椅的实时控制, 如图1所示。 


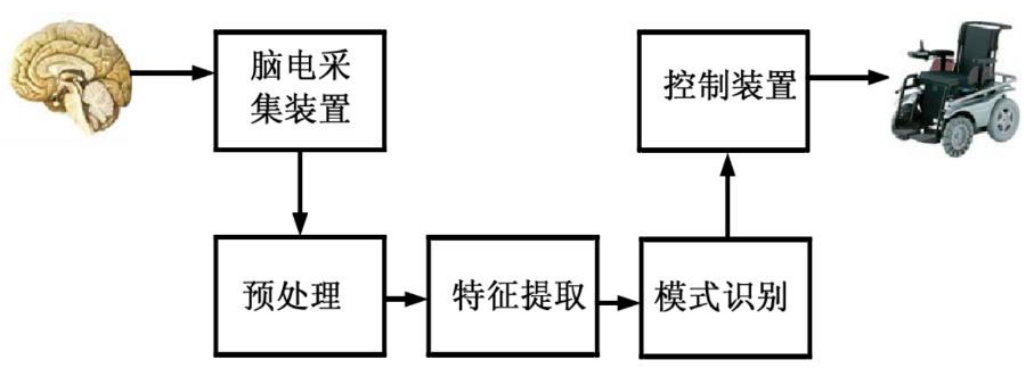

图 1 系统框图

\section{3. 系统的硬件设计}

轮椅终端是本系统的主要识别机构, 主要分为两大模块。 首先是脑电波采集模块, 即TGAM芯片。其主要实现的功 能包括: 与脑电采集电极EEG、REF相连, 再将 TX、RX 端口分别连接到串口蓝牙传输模块的 RX、TX上对原始脑
电波信号进行采集, 并处理和输出 $\alpha 、 \beta$ 等脑波波段数 据。然后是轮椅智控模块, 如图2所示。即核心控制板 S3C6410分别与串口蓝牙模块以及电机驱动等模块进行 相连, 再将电机驱动模块连接到轮椅的电机部分, 分析所 得嵌入式的脑电信号质量, 并发出一些对异常状态的警告 信息（如接触不良或是完全没接触等）, 轮椅则基于普通 电动轮椅, 其相关机械原理在此省略。

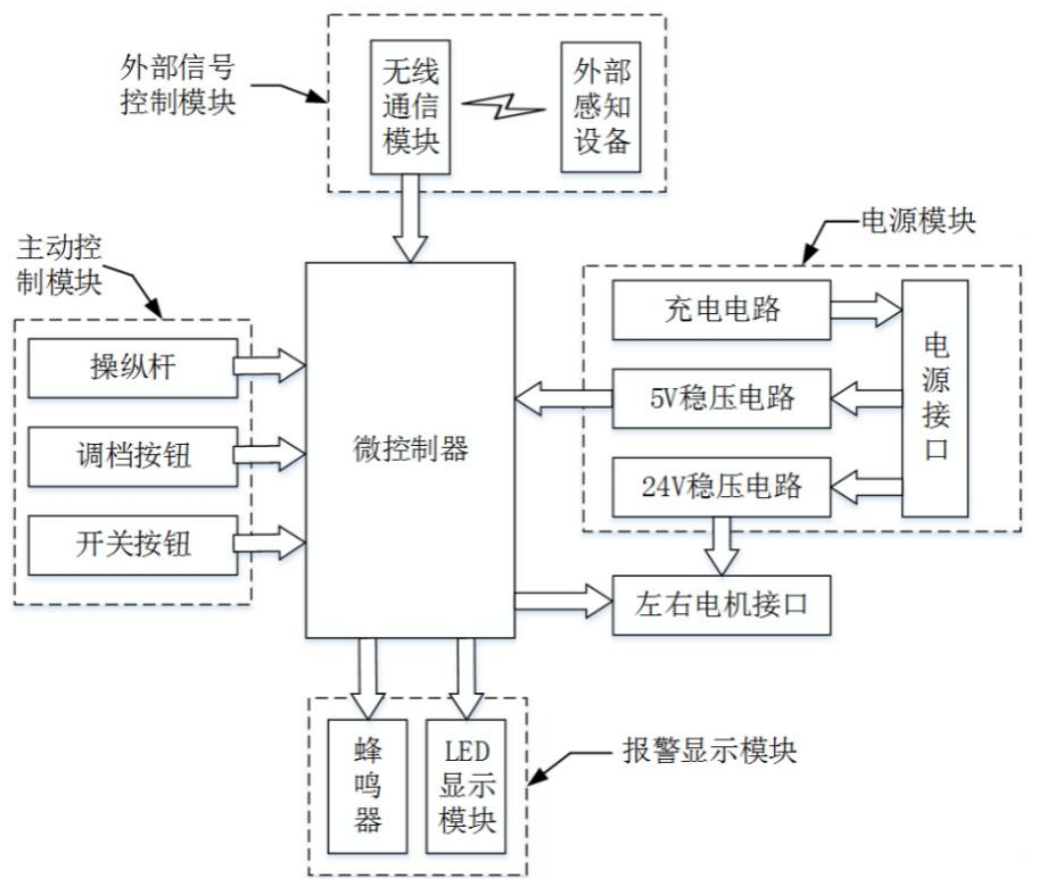

图 2 轮椅控制器结构框图

\section{4. 系统的软件设计}

\section{1. 脑电信号概述}

脑电信号是随机性强的微弱生理电信号, 具有多种节律种 类, 不同个体之间以及同一个体在不同情绪、心态下的脑 电信号都会有显著差异[12]。在兴奋、昏睡、紧张等不同 生理和心理状态下, 人脑电波的频率也不尽相同, 但都大 致分布在 $1-40 \mathrm{~Hz}$ 间。人脑波根据不同频率可以划分成 $\alpha$ 、 $\beta 、 \delta 、 \theta$ 等波。当人在一定的压力下精神高度集中时, 脑波频率在 $12-38 \mathrm{~Hz}$ 之间, 这个波段是 “意识” 层面的脑 波, 即 $\beta$ 波; 当注意力下降, 人进入放松状态时, 脑波 频率会降低到 8-12Hz, 此时为 $\alpha$ 波; 进入睡眠状态后,
脑波频率又进一步下降，又可分为 $\theta$ 波 $(4-8 \mathrm{~Hz})$ 与 $\delta$ 波 ( $0.5-4 \mathrm{~Hz})$ ，反映了人分别在 “潜意识” 和 “无意识” 层面的相关状态。

\section{2. 脑电信号的采集}

本系统中脑电信号采集设备使用的是 NeuroSky 公司开发 的系列 EEG 传感器。该传感器包含以耳垂处电位作为参 考电位的前额传感器, 并且内置了具有自校验采集算法和 高灵敏度放大器的 TGAM 电子芯片, 以数字的方式 (EEG) 来计算电势并消除硬件干扰、帮助噪音过滤。该 系统装置还具有体积小、设置和操作简单、能耗低等优 良的特性。 


\section{3. 基于小波包变换的脑电信号降噪}

小波包变换是一种时频分析方法, 可以将信号按任意时频 分辨率分解到不同的频段 [13], 并且能对信号的高频部分 做更加精细的刻画, 在生物医学信号的提取及去噪处理上 有着广泛应用。

考虑到小波变换在处理信号时相关阈值函数难以选取的 问题, 本系统决定采用小波包变换来实现对原始人体运动 想象脑电采样信号的降噪。在脑电信号的降噪处理中进行 小波包变换主要包含以下几步:

第一步, 先对给定的脑电波信号结合小波正交滤波器进行 小波包变换, 并获得树状小波包的相关参数 [14]。本文选 取了 $\mathrm{db} 4$ 小波滤波器作为实验正交滤波器。

第二步, 采用自底向顶的方法, 根据相应的信息代价函数 并利用最佳小波包基选取算法, 最终搜索出可高效表示所 分析信号的最佳小波包基。

第三步, 利用获得的最佳正交小波包基对应的小波包系数 进行相关的移除噪声处理。

第四步，重新表示降噪后的脑电波信号。

实验表明小波包变换方法能有效地过滤掉和脑电信号混 杂在一起的噪声信号, 并且对 $40 \mathrm{~Hz}$ 之后的噪声信号拥有 优良的抑制效果, 而且对系统的运行效果的影响也不是很 大。

\section{4. 脑电信号特征提取}

脑电信号的特征提取是智能轮椅中脑机接口系统的重要 组成部分。脑电信号的特征提取就是要将收集记录的脑电 信号转换成可以表达不同意识任务的特征向量, 并为分类 器提供最优的输入 [15-16], 从而为后续的模式分类做准 备。出于对相关特征提取算法对正确选取目标特征向量的 高度要求的综合考虑, 为尽量减少计算量、提高信号处理 速度, 本文选取了以下四种与信号能量值相关性较大的时 域特征作为模式分类的特征向量[17], 主要步骤如下:

第一步，计算波长 $W L$ :

$$
W L=\frac{1}{N} \sum_{i=0}^{N-1}\left|x_{i+1}-x_{i}\right|
$$

第二步, 计算平均绝对值 $M A V$ :

$$
M A V=\frac{1}{N} \sum_{i=1}^{N}\left|x_{i}\right|
$$

$N$ 为某通道 $s E M G$ 采样的长度, $i=1,2, \cdots, N, x_{i}$ 为 第 $i$ 点信号的振幅值。

第三步, 计算均值平方根 $R M S$ :

$$
R M S=\sqrt{\frac{1}{N} \sum_{i=1}^{N}\left|x_{i}\right|^{2}}
$$

第四步, 计算脑电波方差值 $V A R$ :

$$
V A R=\frac{1}{N} \sum_{i=0}^{N}\left(x_{i}-\bar{x}\right)^{2}
$$

\section{5. 基于二叉树 $S V M$ 的意念动作模式识别}

系统采用如图3所示的聚类二叉树SVM作为在线训练方 法, 以获取特征向量的值和大量实验结果。图中前进, 后 退, 停止, 左转, 右转分别代表想象相应动作下的脑电波 信号:

(1) 将 (前进+停止+后退) 划分为一类, (左转 + 右转) 划分为一类, 训练出SVM1。

(2) 将 (前进+后退) 划分为一类, (停止) 为一 类, 训练出 SVM2; 左转划分为一类, 后退为一类, 训练 出SVM3。

(3) 前进为一类, 后退为一类, 训练出SVM4。 完成二叉树SVM训练后, 可提取样本降维后的特征向量, 并代入此二叉树SVM进行模式识别。通过测试，可以确 认该分界面的可区分度。在样本模式识别的过程中，应先 从 SVM1开始逐层向下进行，当符号函数为正即停止，同 时得到相应的分类结果。 


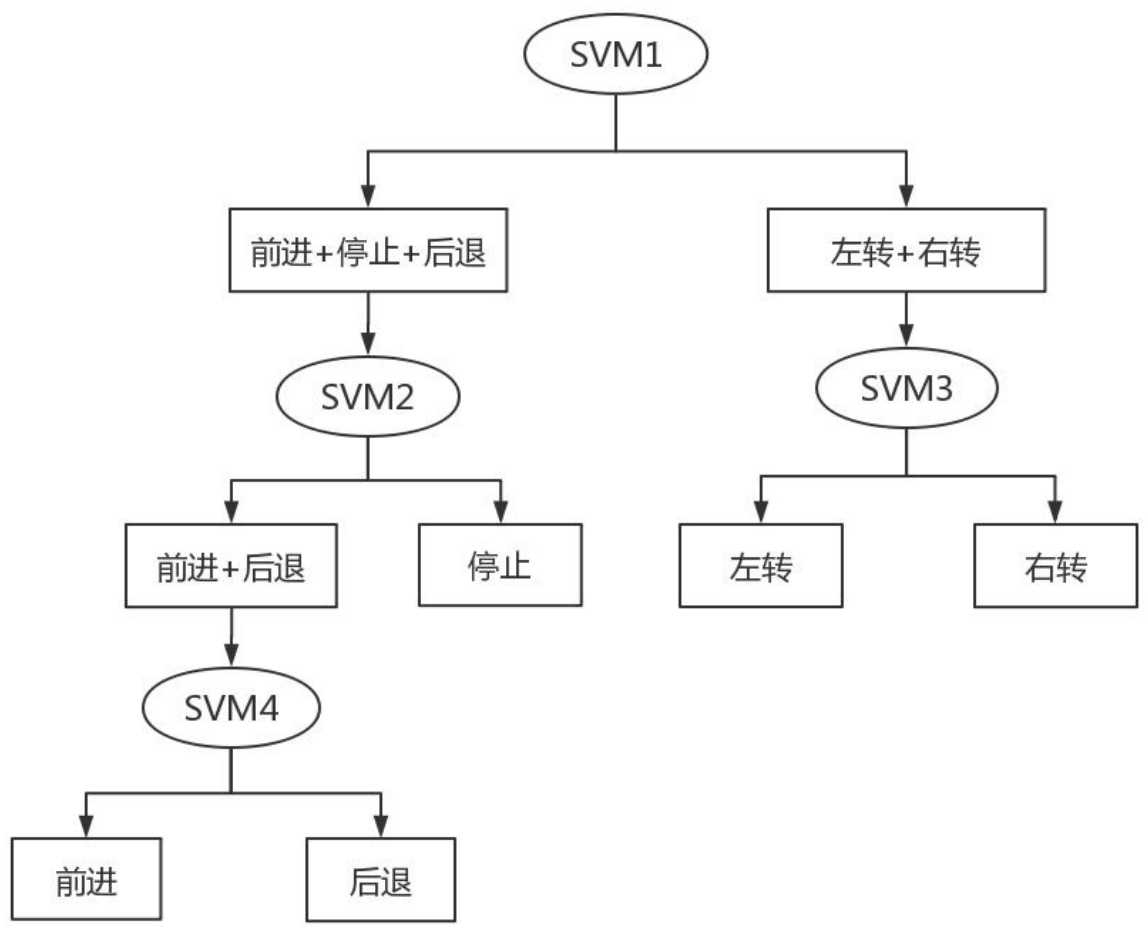

图 3 二叉树 SVM 聚类图

\section{5. 实验结果}

为了验证系统的实用性, 本文先依照上述训练方法, 对某 一志愿者的五种意念动作状态各进行 50 次的预训练, 建 立系统识别模型。之后随机执行每个动作各 50 次, 测试系 统输出的识别结果, 并进行统计。最终的模式识别结果如 表1所示。根据BP神经网络和SVM分类的结果对比证明了 SVM, 能提升分类的准确性和分类精度, 更准确地识别 出用户意图, 从而在脑电信号分类中更具优势。另外, 表 1 中的 5 类意念状态下脑电信号识别结果的平均Kappa系 数达到了 0.622 , 说明意念想象时脑电信号的变化具有一 定的可区分度。因此, 本文设计的系统可以有效识别不同 运动想象状态下的脑电信号, 并能够根据意念控制智能轮 椅的相关运动轨迹, 具有实际应用价值。

表 15 组意念动作识别的 Kappa 值

\begin{tabular}{lccccc}
\hline & 前进 & 后退 & 停止 & 左转 & 右转 \\
\hline BP 神经网络 & 0.55 & 0.52 & 0.59 & 0.58 & 0.53 \\
二叉树 SVM & 0.65 & 0.58 & 0.66 & 0.63 & 0.59 \\
\hline
\end{tabular}

\section{6. 结论}

为了提高肢体功能缺失人士的生活自理能力, 本文设计并 实现了一种基于脑电控制的多功能残疾轮椅系统。系统采 用小波包变换对脑电信号进行降噪并构建时域特征向量, 利用基于二叉树 SVM 分类算法进行人脑运动想象电波的 模式识别。最后将想象不同意念动作的人体脑电信号作为 控制源, 并结合脑电信号的特性实现了实时控制电动轮椅
前进、后退、停止、左转、右转五种运动状态的功能。实 验结果的平均 Kappa 系数达到了 0.622 , 高于传统 BP 神 经网络算法, 进而验证了本文所提智能轮椅人机交互方法 和可靠性以及系统实现方案的有效性。脑电信号控制智能 轮椅的研究已然成为当下工程领域的学术研究热点。本系 统今后的研究工作主要包括:（1）将每个子模块详细设 计后集成在一块硬件板上, 优化系统结构框架, 提高硬件 电路的集成性; (2) 研究提升脑电信号信噪比的有效方 法，使脑电信号具有更高的可靠性和实用性; （3）构建 最优二叉树, 提高系统模式识别的准确率, 探索更有效的 分类方法。

\section{致谢}

本文为地方高校国家级大学生创新创业训练计划基金项 目《基于脑电的智能轮椅控制研究》(编号: 201610412017) 和江西中医药大学重点学科青年教师培养计划基金项目 《卓越模式下医疗电子类专业实验设计》（编号: 2013jzzdxk061）的阶段性成果之一。

\section{参考文献}

[1] Tanaka K, Matsunaga K, Wang H.Electroencepha-log ram-based control of an electric wheelchair[J].IEEE Tran s Robotics, 2005, 21 (4) :762-766.

[2] Iturrate I, Antelis J, K"ubler A, et al.Non-invasivebr ain-actuated wheelchair based on a P300neurophys-iologi cal protocol and automated navigation[J].IEEETrans Rob ot, 2009, 25 (3) :614-627.

[3] Rebsamen B, Guan G T, Zhang H H, et al.A brainc ontrolled wheelchair to navigate in familiar environ-ment 
s[J].IEEE Trans Neural Syst Rehabil Eng, 2010, 18 (6) : 590-598.

[4] Lopes A C, Pires G, Vaz L, et al.Wheelchair naviga -tion assisted by human-machine shared-control and aP3 00-based brain computer interface[C].IntelligentRobots an d Systems.San Francisco:IEEE, 2011:2438-2444.

[5] Gurkan Kucukyildiz,Hasan Ocak,Suat Karakaya,Omer Sayli. Design and Implementation of a Multi Sensor B ased Brain Computer Interface for a Robotic Wheelchair [J]. Journal of Intelligent \& Robotic Systems,2017,87(2). [6] Wang Ker-Jiun,Zhang Lan,Luan Bo,Tung Hsiao-Wei, Liu Quanfeng,Wei Jiacheng,Sun Mingui,Mao Zhi-Hong. Brain-computer interface combining eye saccade two-ele ctrode EEG signals and voice cues to improve the man euverability of wheelchair.[J]. IEEE ... International Conf erence on Rehabilitation Robotics : [proceedings],2017,2 017.

[7] Damodar Reddy Edla,Md Fahim Ansari,Nikhil Chau dhary,Shubham Dodia. Classification of Facial Expressio ns from EEG signals using Wavelet Packet Transform a nd SVM for Wheelchair Control Operations $[\mathrm{J}]$. Procedia Computer Science,2018,132.

[8] NAKAHARA Teiyu,MATSUO Yoshiki,UENO Yuuki. Extraction of the Movement Intention of Legs Using C onsumer EEG Device[J]. The Proceedings of JSME ann ual Conference on Robotics and Mechatronics (Robome c),2016,2016(0).

[9] 涂建成. 脑电信号控制智能轮椅的研究 [D]. 杭州电子 科技大学, 2011 .

[10] 谭筠梅, 蔡子莹. 基于脑电波传感器的智能轮椅设计 [J].自动化与仪器仪表,2015(11):238-240.

[11] Nobuaki Kobayashi,Masahiro Nakagawa. BCI - base d control of electric wheelchair using fractal characterist ics of EEG[J]. IEEJ Transactions on Electrical and Elect ronic Engineering,2018,13(12).

[12] 李颖洁, 邱意弘, 朱贻盛. 脑电信号分析方法及其应 用[M]. 科学出版社, 2009 .

[13] 曾翔,唐广. 基于 EEG 的便携式脑机接口设备研究 $[\mathrm{J}]$. 数字技术与应用,2011(11):41.

[14] 刘珑, 李胜,王轶卿. 基于小波包变换的脑电波信号降 噪及特征提取 [J]. 计算机工程与科学,2015,37(04):790-795.

[15] 施锦河, 沈继忠, 王攀. 四类运动想象脑电信号特征提 取与分类算法 [J].浙江大学学报(工学版),2012,46(02):338344.

[16] 白惠熔,刘旭, 吴佳桐.采用Arduino 平台脑电控制轮椅 运动的研究 [J].科技传播,2016,8(06):83+105.

[17] Nadzri A A B A , Ahmad S A , Marhaban M H , et al. Characterization of surface electromyography usin $\mathrm{g}$ time domain features for determining hand motion an d stages of contraction[J]. 2014. 